\title{
Randomized phase III study of whole-brain radiotherapy for primary CNS lymphoma
}

Korfel, A ; Thiel, E ; Martus, P ; Möhle, R ; Griesinger, F ; Rauch, Mi ; Röth, A ; Hertenstein, B ; Fischer, T ; Hundsberger, T ; Mergenthaler, H G ; Junghanß, C ; Birnbaum, T ; Fischer, L ; Jahnke, K ; Herrlinger, U ; Roth, P ; Bamberg, M ; Pietsch, T ; Weller, M

\begin{abstract}
OBJECTIVE This is the final report of a phase III randomized study to evaluate whole-brain radiotherapy (WBRT) in primary therapy of primary CNS lymphoma (PCNSL) after a median followup of 81.2 months. METHODS Patients with newly diagnosed PCNSL were randomized to high-dose methotrexate (HDMTX)-based chemotherapy alone or followed by WBRT. We hypothesized that the omission of WBRT would not compromise overall survival (OS; primary endpoint), using a noninferiority design with a margin of 0.9. RESULTS In the per-protocol population ( $\mathrm{n}=320)$, WBRT nonsignificantly prolonged progression-free survival (PFS) (median 18.2 vs 11.9 months, hazard ratio [HR] 0.83 [95\% confidence interval (CI) 0.65-1.06], $\mathrm{p}=0.14$ ) and significantly PFS from last HDMTX (25.5 vs 12.0 months, HR 0.65 [95\% CI 0.5-0.83], p = 0.001), but without OS prolongation (35.6 vs 37.1 months, HR 1.03 [95\% CI 0.79-1.35], $\mathrm{p}=0.82)$. In the intent-to-treat population $(\mathrm{n}=410)$, there was a prolongation by WBRT of both PFS (15.4 vs 9.9 months, HR 0.79 [95\% CI 0.64-0.98], p = 0.034) and PFS from last HDMTX (19.4 vs 11.9 months, HR 0.72 [95\% CI 0.58-0.89], p = 0.003), but not of OS (32.4 vs 36.1 months, HR 0.98 [95\% CI 0.79-1.26], $\mathrm{p}=0.98$ ). CONCLUSION Although the statistical proof of noninferiority regarding OS was not given, our results suggest no worsening of OS without WBRT in primary therapy of PCNSL. CLASSIFICATION OF EVIDENCE This study provides Class II evidence that in PCNSL HDMTX-based chemotherapy followed by WBRT does not significantly increase survival compared to chemotherapy alone. The study lacked the precision to exclude an important survival benefit or harm from WBRT.
\end{abstract}

DOI: https://doi.org/10.1212/WNL.0000000000001395

Posted at the Zurich Open Repository and Archive, University of Zurich

ZORA URL: https://doi.org/10.5167/uzh-110459

Journal Article

Accepted Version

Originally published at:

Korfel, A; Thiel, E; Martus, P; Möhle, R; Griesinger, F; Rauch, Mi; Röth, A; Hertenstein, B; Fischer, T; Hundsberger, T; Mergenthaler, H G; Junghanß, C; Birnbaum, T; Fischer, L; Jahnke, K; Herrlinger, U; Roth, P; Bamberg, M; Pietsch, T; Weller, M (2015). Randomized phase III study of whole-brain radiotherapy for primary CNS lymphoma. Neurology, 84(12):1242-1248.

DOI: https://doi.org/10.1212/WNL.0000000000001395 


\section{Randomized phase III study of whole-brain radiotherapy for primary CNS lymphoma}

A. Korfel $M D^{1}, E$. Thiel $M D^{1}, P$. Martus $P^{2} D^{2}, R$. Möhle $M D^{3}, F$. Griesinger $M D^{4}, M$. Rauch $M D^{5}$, A. Röth $M D^{6}$, B. Hertenstein $M D^{7}, T$. Fischer $M D^{8}, T$. Hundsberger $M D^{9}$, H.-G. Mergenthaler $M D^{10}$, Ch. Junghan $M D^{11}$, T. Birnbaum $M D^{12}$, L. Fischer $M D^{1}$, K. Jahnke $M D^{1}$, U. Herrlinger $M D^{13,14}$, P. Roth $M D^{15,16}, M$. Bamberg $M D^{17}$, T. Pietsch $M D^{18}, M$. Weller MD ${ }^{15,16}$

${ }^{1}$ Department of Hematology, Oncology and Tumor Immunology, Charité Berlin, Germany; ${ }^{2}$ Department of Clinical Epidemiology and Applied Biostatistics, University Tuebingen, Germany; ${ }^{3}$ Department of Hematology and Oncology, University Tuebingen, Germany; ${ }^{4}$ Department of Radiotherapy and Medical Oncology, Pius Hospital Oldenburg, Germany; ${ }^{5}$ Department of Neurology, Protestant Hospital Bielefeld, Germany; ${ }^{6}$ Department of Hematology, University Hospital Essen, Germany; ${ }^{7}$ Department of Internal Medicine, Hospital Bremen Center, Germany; ${ }^{8}$ Department of Hematology and Oncology, University Hospital Magdeburg, Germany; ${ }^{9}$ Department of Neurology, University Hospital Mainz, Germany; ${ }^{10}$ Department of Hematology and Oncology, Klinikum Stuttgart, Germany;

${ }^{11}$ Department of Hematology and Oncology, University Hospital Rostock, Germany; ${ }^{12}$ Department of Neurology, University Hospital Munich LMU, Germany; ${ }^{13}$ Department of Neurology, University Hospital Tuebingen, Germany; ${ }^{14}$ Department of Neurology, University Bonn, Germany; ${ }^{15}$ Department of Neurology, University Hospital Tuebingen; Germany; ${ }^{16}$ Department of Neurology, University Hospital Zurich, Switzerland; ${ }^{17}$ Department of Radiation Oncology, University Hospital Tuebingen, Germany; ${ }^{18}$ Institute of Neuropathology, University Hospital Bonn, Germany.

Supplemental data: Table e-1 


\section{Correspondence}

PD Dr. Agnieszka Korfel, Department of Hematology and Oncology, Charité Berlin, Hindenburgdamm 30, D-12200 Berlin, Germany, Tel. +49 30 84454096, Fax +49 30 84452873, E-Mail agnieszka.korfel@charite.de

Word count of the abstract 244

Word count of the paper $\quad 1417$

Character count of the title: $\quad 80$

Number of references: $\quad 12$

Number of figures: $\quad 3$

The statistical analysis was conducted by Peter Martus, Department of Clinical Epidemiology and Applied Biostatistics, University Tuebingen, Germany

Search terms: Randomized controlled, Primary brain tumor, Chemotherapy, Radiation therapy 
A. Korfel designed and wrote the study protocol, contributed patients to the trial, wrote and approved the report.

E. Thiel designed and wrote the study protocol, contributed patients to the trial, approved the report.

P. Martus did the statistical analyses, wrote and approved the report.

R. Möhle contributed patients to the trial, approved the report.

F. Griesinger contributed patients to the trial, approved the report.

M. Rauch contributed patients to the trial, approved the report.

A. Röth contributed patients to the trial, approved the report.

B. Hertenstein contributed patients to the trial, approved the report.

T. Fischer contributed patients to the trial, approved the report.

T. Hundsberger contributed patients to the trial, approved the report.

H.-G. Mergenthaler contributed patients to the trial, approved the report.

Ch. Junghanß contributed patients to the trial, approved the report.

T. Birnbaum contributed patients to the trial, approved the report.

L. Fischer contributed patients to the trial, approved the report.

K. Jahnke contributed patients to the trial, approved the report.

$U$. Herrlinger designed and wrote the study protocol. contributed patients to the trial, approved the report.

P. Roth contributed patients to the trial, approved the report.

M. Bamberg designed and wrote the study protocol, was responsible for central review of radiation oncology, approved the report.

T. Pietsch was responsible for central review of neuropathology, approved the report.

M. Weller designed and wrote the study protocol, contributed patients to the trial, wrote and approved the report.

All authors have no relevant disclosures.

Study funding: Supported by the German Cancer Aid grant 70-2838-Th 


\section{Abstract}

Objective: This is the final report of a phase III randomized study to evaluate the role of whole-brain radiotherapy (WBRT) in primary therapy of primary CNS lymphoma (PCNSL) after a median follow-up of 81.2 months.

Methods: Patients with newly diagnosed PCNSL were randomized to high-dose methotrexate (HDMTX) - based chemotherapy (CHT) followed by WBRT or chemotherapy (CHT) alone. We hypothesized that the omission of WBRT from firstline treatment would not compromise overall survival (OS; primary endpoint), using a non-inferiority design with a margin of 0.9 .

Results: Of 410 patients who entered the post-HDMTX phase (intention-to-treat, ITT population) 320 were treated per-protocol (PP). In the PP-population, there was a non significant prolongation of progression-free survival (PFS) with WBRT: median 18.2 versus 11.9 months, HR $0.83(95 \% \mathrm{Cl} 0.65-1.06), \mathrm{p}=0.14$, and a significant prolongation of PFS from last HDMTX: 25.5 versus 12.0 months, $\mathrm{HR} 0.65(95 \% \mathrm{Cl}$ 0.5-0.83), $\mathrm{p}=0.001$, but no OS prolongation: 35.6 versus 37.1 months, HR 1.03 $(95 \% \mathrm{Cl} 0.79-1.35), \mathrm{p}=0.82$. The results in the ITT population were similar to those in the PP population with prolongation by WBRT of both PFS: 15.4 versus 9.9 months, HR 0.79 (95\% Cl 0.64-0.98), $\mathrm{p}=0.034$, and PFS from last HDMTX: 19.4 versus 11.9 months, HR $0.72(95 \% \mathrm{Cl} 0.58-0.89), \mathrm{p}=0.003$, but not of OS: 32.4 versus 36.1 months, HR 0.98 (95\%Cl 0.79-1.26), $\mathrm{p}=0.98$.

Conclusion: Although the statistical proof of non-inferiority regarding OS was not given our results suggest no worsening of OS without WBRT in primary therapy of PCNSL. 
Classification of evidence: This study provides Class I evidence that WBRT can be omitted in primary treatment of PCNSL. 


\section{Introduction}

Standards of care have not been well defined for primary CNS lymphoma (PCNSL). High-dose methotrexate (HDMTX) is the only undisputed standard of care whereas the addition of whole-brain radiotherapy (WBRT) has been increasingly questioned because of the high frequency of late neurotoxicity after combined modality treatment. In 1999, the German PCNSL Study Group (G-PCNSL-SG) had designed a prospective, randomized trial to test the hypothesis that primary HDMTX-based chemotherapy $(\mathrm{CHT})$ alone was not inferior to primary $\mathrm{CHT}$ followed by WBRT for immunocompetent patients with newly diagnosed PCNSL. The 2010 report of overall survival (OS; the primary endpoint) at a median follow-up of 31.8 months had resulted in the conclusion that WBRT can be omitted from first-line treatment of PCNSL without compromising OS although non-inferiority had not been formally proven. ${ }^{1}$ Here, we present updated and final data including a new "as-treated" analysis after a median follow-up of 81.2 months.

\section{Patients and Methods}

\section{Standard Protocol Approvals, Registrations, and Patient Consents}

The study was approved by the local Human Investigations Committee, and informed consent was obtained from each participant. The study is registered with ClinicalTrials.gov, number NCT00153530.

\section{Study Design, Selection of Participants and Interventions}

The G-PCNSL-SG1 included immunocompetent patients with newly diagnosed

PCNSL. ${ }^{1}$ After 1:1 randomization performed at study enrolment all patients were to 
receive HDMTX from 1999-2007 and HDMTX plus ifosfamide thereafter. Patients achieving complete response $(\mathrm{CR})$ received consolidating WBRT or no further treatment, whereas patients without $\mathrm{CR}$ received WBRT or second-line $\mathrm{CHT}$ with high-dose cytarabine (HD-Ara-C).

\section{Study Endpoints and Statistical Analysis}

The goal of the trial was to demonstrate that the omission of WBRT from first-line treatment does not compromise OS as the primary endpoint using a non-inferiority design with a margin of 0.9 . OS was measured from the time of randomization until death; progression-free survival (PFS) was measured until progression or death. Further, to better assess the role of therapy administered after HDMTX-based CHT (HD-Ara-C or WBRT), an additional PFS calculation was performed from the time of last HDMTX-based $\mathrm{CHT}$ to progression or death per response group (CR versus partial remission (PR), stable disease (SD) and progressive disease (PD) pooled) herein referred to as PFS from last HDMTX. Additionally, we analysed the ITT population by the treatment that was actually given ("as-treated") e.g. patients randomized to WBRT who did not receive WBRT (and received second-line CHT instead or no further therapy) were analyzed as no WBRT and patients randomized to no WBRT who received WBRT were analyzed as WBRT. As the consequence, patients with CR after HDMTX-based CHT were divided in two groups: WBRT and no WBRT (= no further therapy) whereas non-CR patients were divided in three groups: WBRT, CHT and no therapy (patients who were left with PR, SD or PD after HDMTX-based CHT without any further therapy). Multivariate analysis was performed by using the Cox proportional hazard model. All hazard ratios refer to the 
comparison of WBRT vs. no WBRT i.e. hazard ratios lower than 1 are in favour of WBRT.

\section{Results}

From 1999-2009, 551 patients (median age: 63 years) were enrolled; of those, 524 fulfilled the eligibility criteria and started HDMTX-based primary CHT (primary eligibility population), 410 entered the post-HDMTX phase with a known response status (ITT population) and 320 were treated as randomized (PP population) ${ }^{1}$ (Fig 1). The median follow up was 81.2 months.

In the PP population, there was a non significant PFS prolongation with WBRT: median 18.2 versus 11.9 months, HR $0.83(95 \% \mathrm{Cl} 0.65-1.06), \mathrm{p}=0.14$, and a significant prolongation of PFS from last HDMTX: 25.5 versus 12.0 months, HR 0.65 (95\% Cl 0.5-0.83), $\mathrm{p}=0.001$. In contrast, early WBRT did not prolong OS: 35.6 versus 37.1 months, HR $1.03(95 \% \mathrm{Cl}$ 0.79-1.35), $\mathrm{p}=0.82$.

When $\mathrm{CR}$ and non-CR patients were considered separately, any potential positive impact of WBRT was more prominent in non-CR patients. In CR patients, a nonsignificant prolongation of PFS and PFS from last HDMTX were found with WBRT: 42.5 versus 22.3 months, $\mathrm{HR} 0.69(95 \% \mathrm{Cl} 0.46-1.03), \mathrm{p}=0.065$; and 40.1 versus 19.1 months, HR 0.68 (95\% Cl 0.46-1.01), $\mathrm{p}=0.057$, respectively. There was no difference in OS: 44.2 versus 59.0 months, $\mathrm{HR} 1.06(95 \% \mathrm{Cl} 0.69-1.63), \mathrm{p}=0.78 . \mathrm{In}$ non-CR patients, both PFS and PFS from last HDMTX were prolonged with WBRT: 5.0 versus 2.9 months, $\mathrm{HR} 0.6(95 \% \mathrm{Cl} 0.43-0.83), \mathrm{p}=0.002$; and 16.1 versus 2.9 months, HR 0.41 (95\% Cl 0.29-0.57), p<0.001; respectively, whereas no difference 
in OS was found: 27.4 versus 18.2 months, $\operatorname{HR~} 0.76(95 \% \mathrm{Cl} 0.54-1.08), p=0.119$ (Fig 2A).

The results in the ITT population were similar to those in the PP population with prolongation by WBRT of both PFS: 15.4 versus 9.9 months, HR 0.79 (95\% Cl 0.640.98), $\mathrm{p}=0.034$, and PFS from last HDMTX: 19.4 versus 11.9 months, HR 0.72 (95\% $\mathrm{Cl} 0.58-0.89), p=0.003$, but not of OS: 32.4 versus 36.1 months, $\mathrm{HR} 0.98(95 \% \mathrm{Cl}$ 0.79-1.26), $p=0.98$. In CR patients, no differences in PFS, PFS from last HDMTX and OS were found: 29.9 versus 25.7 months, HR 0.85 (95\% Cl 0.6-1.2), $\mathrm{p}=0.35$; 27.8 versus 23.4 months, $\mathrm{HR} 0.84(95 \% \mathrm{Cl} 0.6-1.19), \mathrm{p}=0.33$ and 51.3 versus 61.0 months, HR 1.13 (95\% Cl 0.77-1.66), $\mathrm{p}=0.53$, respectively. In non-CR patients there was a prolongation of both PFS: 4.7 versus 2.9 months, HR 0.67 (95\% Cl 0.51-0.89), $\mathrm{p}=0.004$, and PFS from last HDMTX: 15.5 versus 5.7 months, $\mathrm{HR} 0.58(95 \% \mathrm{Cl}$ 0.44-0.77), $\mathrm{p}<0.001$ with WBRT, but no difference in OS: 20.7 versus 18.6 months, HR 0.86 (95\% Cl 0.64-1.16), p=0.32, (Fig 2B).

Multivariate analysis revealed lower Karnofsky performance score (KPS) as a risk factor for PFS in both the PP and the ITT population. For PFS from last HDMTX, besides lower KPS, not having received WBRT was a risk factor. For OS, higher age and lower KPS were risk factors in the PP and the ITT population, whereas male gender was a risk factor only in the PP, but not in the ITT population (Suppl Table).

Results of the "as-treated" analysis supported the observations made in the PP and ITT populations (Fig 3). In CR patients PFS from last HDMTX was 33.8 months with WBRT and 19.0 months without; HR 0.64 (95\% Cl 0.44-0.94), $\mathrm{p}=0.025$ and OS 51.9 versus 59.0 months, HR $0.93(95 \% \mathrm{Cl} 0.68-1.53), \mathrm{p}=0.95$. In non-CR patients PFS from last HDMTX was 15.9 months with WBRT, 3.2 months with $\mathrm{CHT}$ and 8.9 
months without further therapy, HR 0.47 (95\% Cl 0.35-0.62), p<0.001; OS was 23.8, 14.8 and 27.5 months, respectively, $\operatorname{HR} 0.76(95 \% \mathrm{Cl} 0.56-1.02), \mathrm{p}=0.172$, respectively.

\section{Discussion}

Long-term results of the G-PCNSL-SG1 trial suggest that there is no significant difference in OS when WBRT is omitted from the first-line treatment of PCNSL. Yet, the primary hypothesis of non-inferiority according to the study protocol which asked for a lower confidence limit of 0.9 was not proven. There was, however, a significant PFS prolongation with WBRT both in the PP and ITT population (in the PP population of PFS from last HDMTX only), which was most prominent in non-CR patients.

G-PCNSL-SG-1 may thus define a role of WBRT for disease control in PCNSL. WBRT was more effective than HD-Ara-C in patients with disease less- or insensitive to HDMTX. This did not translate into a significant OS benefit with only a trend towards longer OS in non-CR patients, most probably because of the effectiveness of other salvage treatments. However, the study was not powered to detect differences in CR and non-CR patients evaluated separately. Compromised PFS, but not OS by deferring WBRT has also been observed in two recent retrospective analyses. ${ }^{2,3}$

Late neurotoxicity is the principal reason to withhold WBRT from initial therapy of PCNSL. In the G-PCNSL-SG1 trial clinical and radiologic signs of late neurotoxicity were found more frequently in patients after WBRT. ${ }^{1}$ This is in accordance with the results of a retrospective analysis which identified WBRT as the only factor significantly associated with late neurotoxicity. ${ }^{4}$ Moreover, in two recent analyses 
using detailed neuropsychologic testing more cognitive dysfunction was observed with HDMTX-based CHT plus WBRT than with $\mathrm{CHT}$ alone., ${ }^{5,6}$ Although elderly patients are considered at particular risk ${ }^{7}$, clinically manifest late neurotoxicity was also found in $>20 \%$ of younger patients after combined $\mathrm{CHT}_{\mathrm{NBRT}}^{8,9}$ and even in $63 \%$ of patients when extensive neuropsychological assessment was used. ${ }^{10}$ Whether reduced-dose WBRT is save or necessary for improved outcome or both ${ }^{11}$ requires a randomized trial such as RTOG 1114 (NCT 01399372).

Based on our results, WBRT can be postponed until relapse in patients with CR to HDMTX-based $\mathrm{CHT}$ in younger patients. Conversely, in patients aged 60 years or older who are at increased risk of both late neurotoxicity and relapse ${ }^{12}$, alternative strategies of CR consolidation must be explored.

\section{Acknowledgment}

The authors thank Dieter Augustin for his excellent assistance in data analysis and Brigitta Rieger for her help in data collection.

\section{References}

1. Thiel E, Korfel A, Martus P, et al. High-dose methotrexate with or without brain radiotherapy for primary CNS lymphoma (G-PCNSL-SG1): a phase 3, randomised, non-inferiority trial. Lancet Oncology 2010;11(11):1036-1047.

2. Omuro A, Taillandier L, Chinot $\mathrm{O}$, et al. Primary CNS lymphoma in patients younger than 60: can whole-brain radiotherapy be deferred? J Neurooncol 2011;104(1):323-330. 
3. Ekenel M, Iwamoto FM, Ben-Porat LS, et al. Primary central nervous system lymphoma: the role of consolidation treatment after a complete response to highdose methotrexate-based chemotherapy. Cancer. 2008;113(5):1025-1031.

4. Omuro AM, Ben-Porat LS, Panageas KS, et al. Delayed neurotoxicity in primary central nervous system lymphoma. Arch Neurol. 2005;62(10):1595-1600.

5. Correa DD, Shi W, Abrey LE, et al. Cognitive functions in primary CNS lymphoma after single or combined modality regimens. Neuro Oncol 2012.;14(1):101-108.

6. Doolittle ND, Korfel A, Lubow $M$, et al. Long-term cognitive function, neuroimaging and quality of life in primary CNS lymphoma. Neurology 2013;81(1):84-92

7. Abrey LE, Yahalom J, DeAngelis LM. Treatment for primary CNS lymphoma: the next step. J Clin Oncol. 2000;18(17):3144-3150.

8. Gavrilovic IT, Hormigo A, Yahalom J, et al. Long-term follow-up of high-dose methotrexate-based therapy with and without whole brain irradiation for newly diagnosed primary CNS lymphoma. J Clin Oncol. 2006;24(28):4570-4574. 
9. Kasenda B, Schorb E, Fritsch K, et al. Prognosis after high-dose chemotherapy followed by autologous stem-cell transplantation as first-line treatment in primary CNS lymphoma--a long-term follow-up study. Ann Oncol 2012;23(10):2670-2675.

10. Harder $\mathrm{H}$, Holte $\mathrm{H}$, Bromberg JE, et al. Cognitive status and quality of life after treatment for primary CNS lymphoma. Neurology 2004;62:544-547.

11. Morris PG, Correa DD, Yahalom J, et al. Rituximab, methotrexate, procarbazine, and vincristine followed by consolidation reduced-dose whole-brain radiotherapy and cytarabine in newly diagnosed primary CNS lymphoma: final results and long-term outcome. J Clin Oncol. 2013;31(31):3971-3979.

12. Roth $\mathrm{P}$, Martus $\mathrm{P}$, Kiewe $\mathrm{P}$, et al. Outcome of elderly patients with primary CNS lymphoma in the G-PCNSL-SG-1 trial. Neurology 2012;79:890-896. 


\section{Figure legends}

Figure 1 Trial profile (CONSORT flow diagram)

Study arm WBRT: Two additional patients were excluded during HDMTX-based $\mathrm{CHT}$ (one with spinal lymphoma, now classified as "did not meet inclusion criteria" and one who refused CHT continuation in two-week intervals, now classified as "refused participation"). For one patient the documentation of 6th HDMTX course and CR achievement was obtained, and he was reclassified as "ITT, with CR, no further treatment". One patient was reclassified by the treating physician as having CR and moved from "ITT, without CR, had no further therapy" to "ITT, CR, refused to receive WBRT".

Study arm no WBRT: One patient was wrongly classified as "lost to follow-up

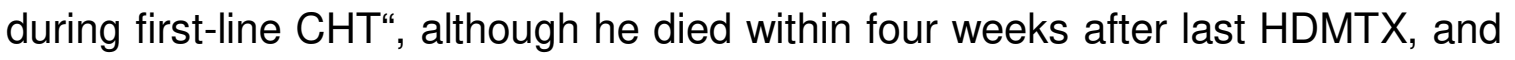
is now classified as "died during first-line $\mathrm{CHT}$ ". In two patients documentation of second-line therapy with HD-Ara-C was obtained, and they were moved from "did not receive HD-Ara-C" to "received HD-Ara-C". In one patient remission status (SD) was changed after review of all scans to CR by the treating physician, and he was moved from "ITT, without CR, received WBRT" to "ITT, with CR, received WBRT".

Abbreviations: $\mathrm{CHT}=$ chemotherapy, $\mathrm{CR}=$ complete response; $\mathrm{HD}-\mathrm{Ara}-\mathrm{C}=$ high-dose cytarabine; ITT = intent-to-treat; PP = per-protocol; SD = stable disease; WBRT = whole-brain radiotherapy. 
Figure 2 OS according to the remission status (CR versus non-CR) after HDMTX based $\mathrm{CHT}$ (A) PP population. (B) ITT population.

Abbreviations: $\mathrm{CHT}=$ chemotherapy $\mathrm{CR}=$ complete response; $\mathrm{HDMTX}=$ high-dose methotrexate; ITT = intent-to-treat population; PP = per-protocol population, WBRT = whole-brain radiotherapy.

Figure 3 PFS from last HDMTX-based CHT and OS analysed as-treated in the ITT population. (A) PFS from last HDMTX-based $\mathrm{CHT}$ in patients with $\mathrm{CR}$. (B) PFS from last HDMTX-based CHT in patients without CR. (C) OS in patients with CR. (D) OS in patients without CR. The good outcome of the non-CR patients without further treatment can be explained by the fact that six of them most probably did in fact have CR after HDMTX-based CHT: they were documented as having CR upon follow-up without further therapy. Moreover, one additional patient received WBRT without progression six months after HDMTX-based CHT.

Abbreviations: $\mathrm{CHT}=$ chemotherapy $\mathrm{CR}=$ complete response; $\mathrm{HDMTX}=$ high-dose methotrexate; ITT = intent-to-treat population; PFS = progression-free survival; $\mathrm{WBRT}=$ whole-brain radiotherapy . 
Figure 1 Trial profile (CONSORT flow diagram)

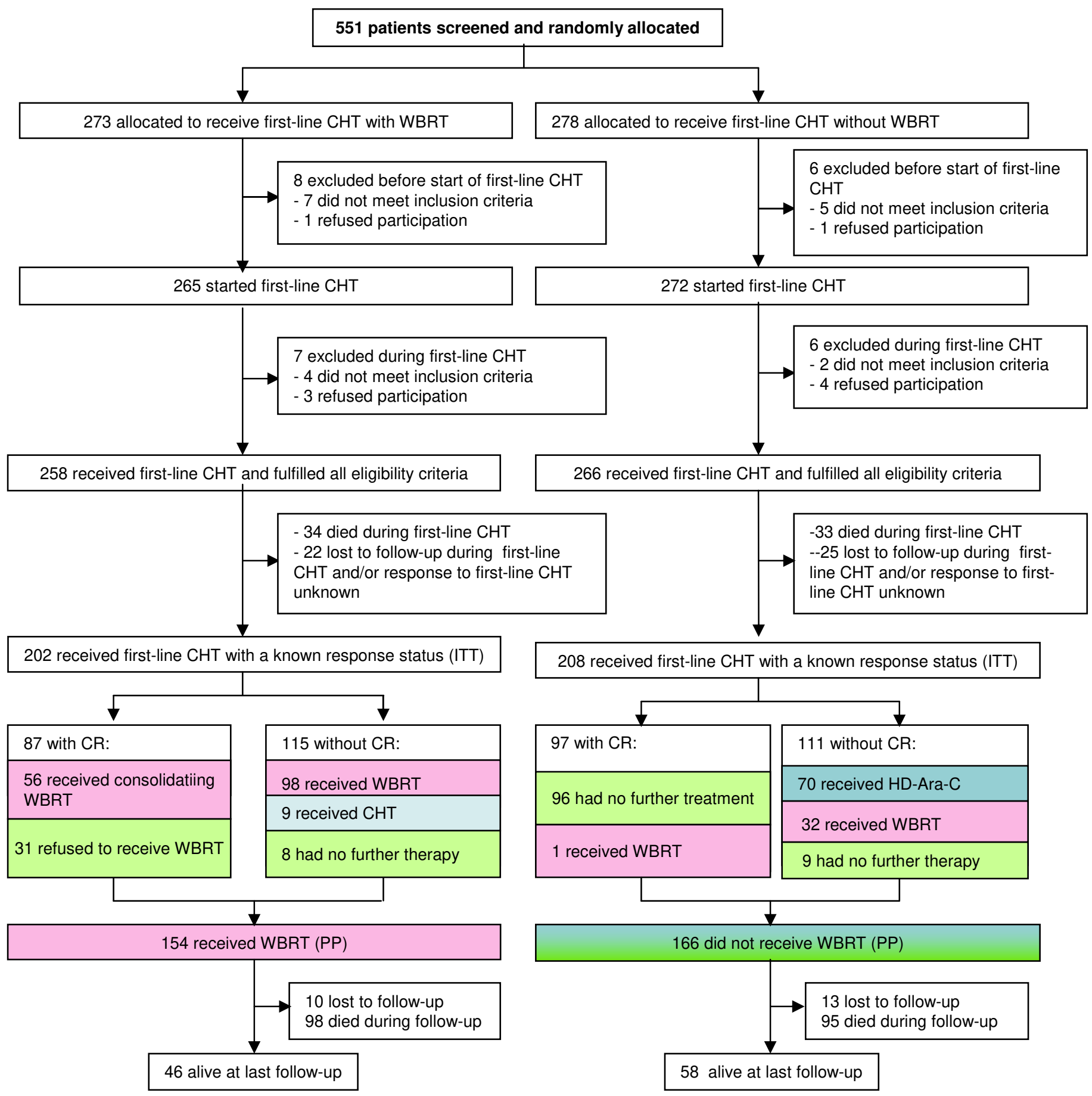

Study arm WBRT: Two additional patients were excluded during HDMTX-based CHT (one with spinal lymphoma, now classified as "did not meet inclusion criteria" and one who refused CHT continuation in two-week intervals, now classified as "refused participation"). For one patient the documentation of 6th HDMTX course and CR achievement was obtained, and he was reclassified as "ITT, with CR, no further treatment". One patient was reclassified by the treating physician as having CR and moved from "ITT, without CR, had no further therapy" to "'ITT, CR, refused to receive WBRT".

Study arm no WBRT: One patient was wrongly classified as "lost to follow-up during first-line CHT", although he died within four weeks after last HDMTX, and is now classified as "died during first-line CHT". In two patients documentation of second-line therapy with HD-Ara-C was obtained, and they were moved from "did not receive HD-Ara-C" to "received HD-Ara-C". In one patient remission status (SD) was changed after review of all scans to CR by the treating physician, and he was moved from "ITT, without CR, received WBRT" to "ITT, with CR, received WBRT".

Abbreviations: $\mathrm{CHT}=$ chemotherapy, $\mathrm{CR}=$ complete response; $\mathrm{HD}-\mathrm{Ara}-\mathrm{C}$ = high-dose cytarabine; ITT = intent-to-treat; $\mathrm{PP}=$ per-protocol; $\mathrm{SD}=$ stable disease; $\mathrm{WBRT}=$ whole-brain radiotherapy . 
Figure 2 OS according to the remission status (CR versus non-CR) after HDMTX-based CHT (A) PP population. (B) ITT population.
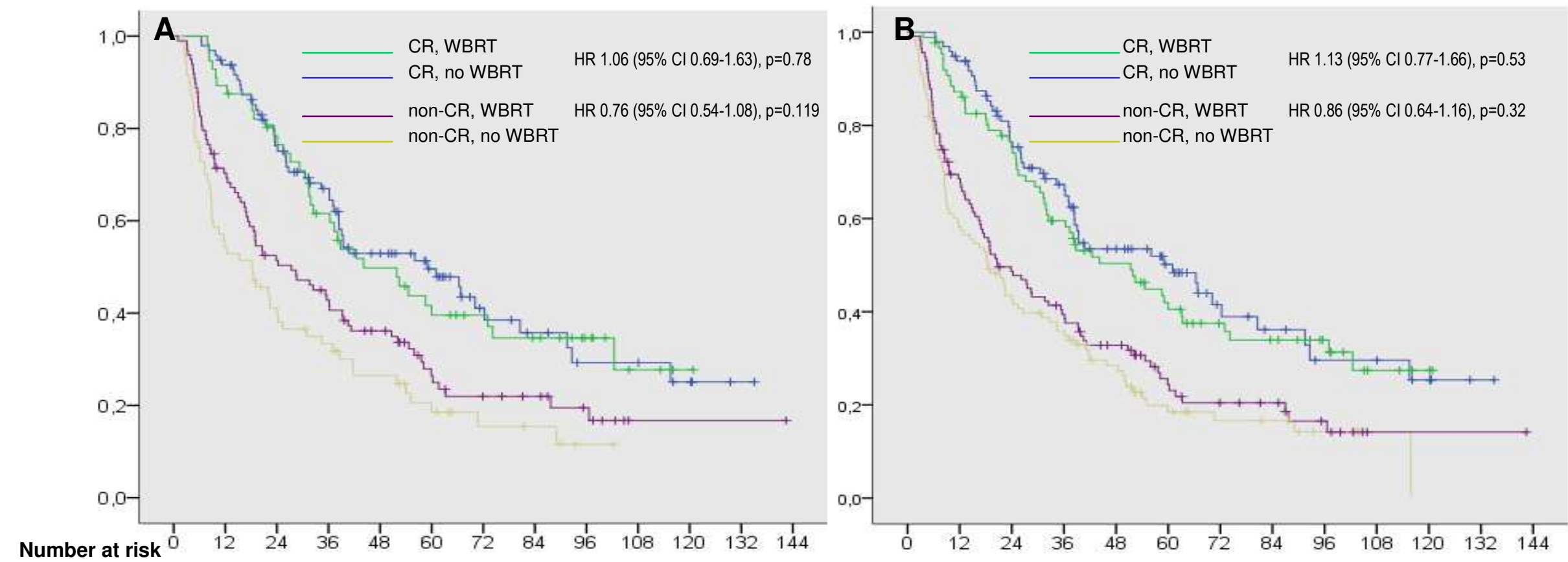

$\begin{array}{llllllllllllll}\text { CR, WBRT } & 56 & 50 & 42 & 32 & 25 & 19 & 16 & 13 & 8 & 3 & 1 & 0 & 0 \\ \text { CR, no WBRT } & 96 & 89 & 68 & 54 & 38 & 28 & 16 & 12 & 8 & 3 & 1 & 1 & 0 \\ \text { non-CR, WBRT } & 98 & 67 & 48 & 39 & 30 & 18 & 13 & 11 & 7 & 1 & 1 & 1 & 0 \\ \begin{array}{l}\text { non-CR, } \\ \text { no WBRT }\end{array} & 70 & 38 & 27 & 21 & 15 & 9 & 5 & 4 & 1 & 0 & 0 & 0 & 0\end{array}$

$\begin{array}{rrrrrrrrrrrrr}87 & 75 & 62 & 47 & 37 & 28 & 21 & 18 & 13 & 5 & 2 & 0 & 0 \\ 97 & 90 & 69 & 55 & 39 & 28 & 16 & 12 & 8 & 8 & 5 & 1 & 0 \\ 115 & 76 & 53 & 42 & 32 & 19 & 14 & 12 & 7 & 1 & 1 & 1 & 0 \\ 111 & 64 & 46 & 37 & 25 & 13 & 9 & 8 & 4 & 1 & 0 & 0 & 0\end{array}$
no WBRT

Abbreviations: $\mathrm{CHT}=$ chemotherapy; $\mathrm{CR}=$ complete response; HDMTX = high-dose methotrexate; ITT = intent-to-treat population; $\mathrm{PP}=$ per-protocol population, WBRT $=$ whole-brain radiotherapy. 
Figure 3 PFS from last HDMTX-based CHT and OS analysed as-treated in the ITT population. (A) PFS from last HDMTX-based CHT in patients with CR. (B) PFS from last HDMTX-based CHT in patients without CR. (C) OS in patients with CR. (D) OS in patients without $\mathrm{CR}$.
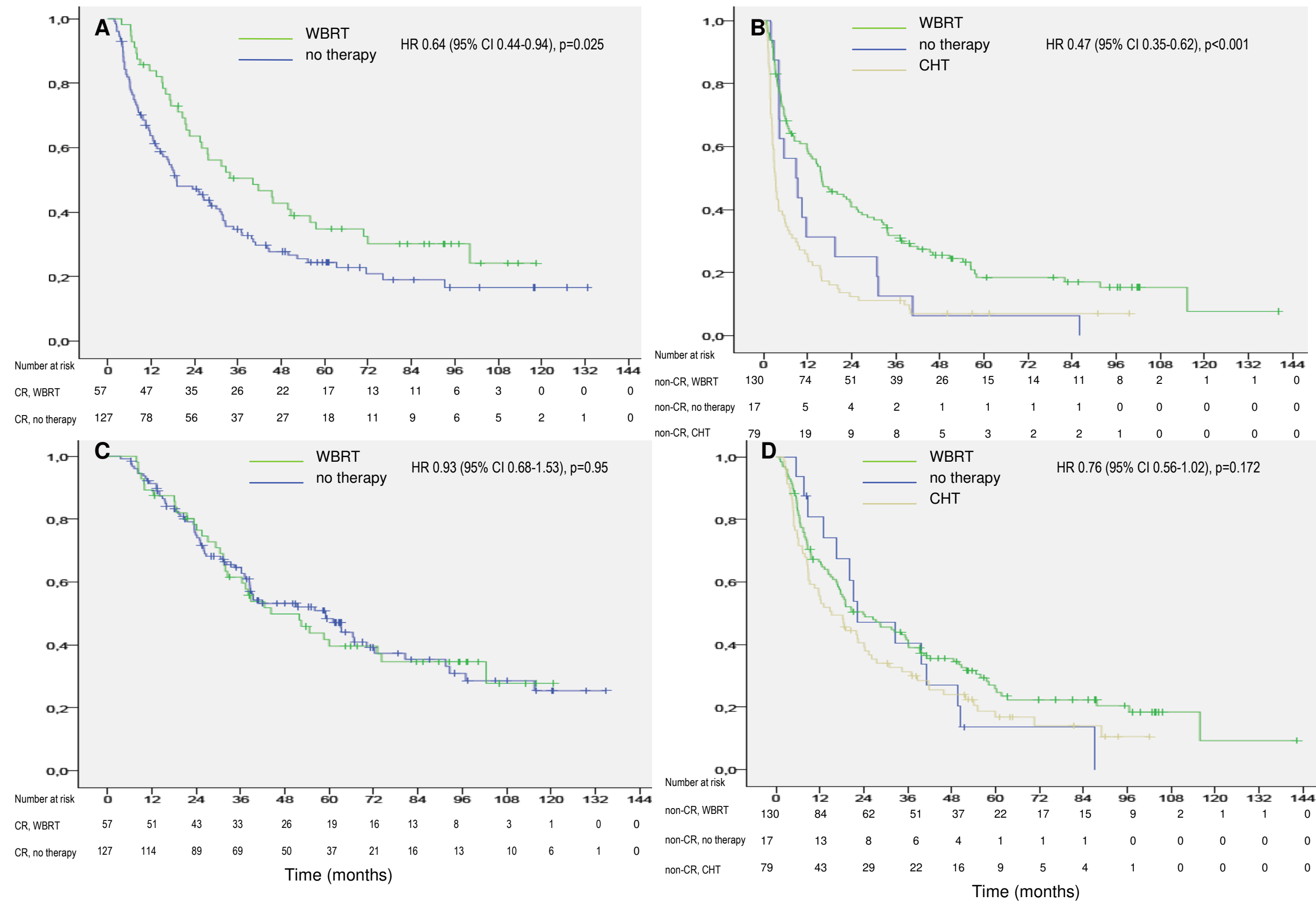
The good outcome of the non-CR patients without further treatment can be explained by the fact that six of them most probably did in fact have CR after HDMTX-based CHT: they were documented as having CR upon follow-up without further therapy. Moreover, one additional patient received WBRT without progression six months after HDMTX-based CHT.

Abbreviations: $\mathrm{CHT}=$ chemotherapy; $\mathrm{CR}=$ complete response; $\mathrm{PFS}=$ progression-free survival; $\mathrm{HDMTX}=$ high-dose methotrexate; ITT = intent-to-treat population; WBRT = whole-brain radiotherapy. 